If my contention in this and previous papers be correct, that residual affinity thus plays a far more important part than has hitherto been supposed, and that it must be taken into account in all discussions on valency, it follows of necessity that our views regarding the constitution of the majority of compounds at present rest upon a most uncertain basis : the constitution of the paraffins, of the benzenes, and of the haloid co.npounds and alcohols derived from the hydrocarbons of these series, may be regarded as determined with a degree of precision almost amounting to certainty; but in the vast majority of other cases we have as yet no secure method of arriving at conclusions which in any sense approach finality. There can be little doubt that in framing our modern conceptions of valency we have been too much influenced by the graphic symbols which have been so widely made use of. In the future it will be necessary to attach a more liberal interpretation to the facts, and it may be hoped that it will some day be possible also to take into account differences depending on the relation of the different forms of matter to the pervading medium.

The properties of compounds being demonstrably dependent on the intramolecular conditions, it is difficult for a chemist to resist the feeling that the peculiarities manifested by the different elements are also very probably the outcome of differences in structure; such an assumption indeed affords at present apparently the only explanation that can be given of the relationship manifest between different elements when these are classified in groups of "homologues" in accordance with the suggestion originally made by Dumas, which has now found full expression in the so-called periodic system of classification. There appears to be an increasing weight of evidence to favour the assumption that the influence exercised by compounds in cases of chemical change is local in its origin : that it is exercised more by a particular constituent or constituents-in particular directions, in fact-than by the molecule as a whole. The suggestion above made that "affinity" acts in particular directions in elementary atoms, and parhaps with different degrees of freedom in various directions, is therefore but an extension to elements of what is more or less generally recognized as the case in com pounds. Some such hypothesis is certainly re quired to account for the existence of allotropic modifications both of non-metals and of metals; for the remarkable changes in magnetic and other properties which iron undergoes with change of temperature; for the different values of the dielectric constant-along the several axes in sulphur crystals ; for the difference in electric conductivity of bismuth in two different directions in bismuth crystals; for the existence of planes in crystals in which cleavage takes place with special readiness, \&c. -all these are instances which apparently afford evidence of atomic dissymmetry. May, not valency after all depend-not in the number of "charges" carried by the atom, but - on the number of directions in which the ever-present "lines of force" are free to act?

\section{WORK OF THE KEW OBSERVATORY IN} I 887 .

IIE Annual Report of the Kew Committee, just issued, show that the activity of the staff of the Kew Observatory is still well sustained, and the various departments devoted to observations-magnetic, meteorological, and solar-verification of scientific apparatus of various kinds, rating of time-pieces, and experiment, all show a considerable turn out of work. In addition to the regular periodical magnetical observations, the main results of which are given in a concise form in the appendixes, assistance was rendered to Profs. Rücker and Thorpe in respect to their valuable magnetic survey of Great Britain, which we are glad to learn they have now completed, after having devoted the greater portion of their vacations to the task for the la it four years. The labours of the Krakatão Committee of the Royal Society, the Magnetic Committee of the British Association, the late Prof. Balfour Stewart, and other investigators, have also been supplemented by aid afforded by the Kew staff.

The meteorological staff have during the year recorded, principaily on behalf of the Meteorological Council, who defray the expenses attendant on the work, some 57, I 26 observations averaging over 150 per diem; the resulting monthly and annual means are, by permission of the Council, published as appendixes.

The multiplication of Observatories engaged in solar photo- graphy at home and abroad having rendered unnecessary the co-operation of kew in that branch of science, so energetically carried on there by the present Chairman, Mr. De la Rue, and the late Prof. Balfour Stewart, twenty years ago, the photoheliograph has only been employed of late years as an ordinary telescope, by means of which the counting of new sunspot groups is continued after Schwabe's method.

An appendix shows that during the last year 44 new groups were catalogued, and that on 60 days out of 180 days of observations the sun's surface was free from spots.

Under the heading "Experimental Work" we find a good deal of attention has been devoted to the photography of high cirrus clouds simultaneously from two points, with the view of determining their position and motions ; and to the question of the proper construction of black bulb thermometers; and also to preparatory operations with the Indian Government pendulum apparatus, preliminary to repeating the observations made at the Observatory by Basevi, Heaviside, and Herschel.

There is a long list of various instruments compared and certified during the year in the verification department, which shows that nearly 14,000 articles belonging to one or the other of twenty-seven different classes have undergone treatment; as instruments newly brought within the influence of the verifier, attention is di ected to range.finders for the use of the Army and Navy, telescopes of the Admiralty pattern, and surveying aneroids.

The popularity of the Kew certificates, as to the time-keeping of watches, shows that the demand for a guarantee as to the accuracy of performance of a watch other than the maker's name actually exists, and no less than 5 Io watches and 27 marine chronometers have been submitted to the rating department since the last report was issued.

An appendix showing the behaviour of the best of the watches during the test is given, and it is found that places in this list are being strongly contested for by watch manufacturers, as the blue ribbons of the trade. In consequence of the growth of the work done at Kew, steps have been taken to obtain the permission of Her Majesty's Chief Commissioner of Works and Public Buildings to enlarge the Observatory, which at present remains almost in the same condition as it stood 130 years ago when originally erected as His Majesty George III.'s private Observatory at Richmond.

\section{THE TOTAL ECLIPSE OF THE MOON, JANUARY 28.}

$\mathrm{BY}$ the kindness of the Astronomer Royal for Scotland, $\mathrm{Mr}$. Gledhill, of Mr. Crossley's observatory at Bermerside, and Mr. Stothert, all of whom took part in the observation of the eclipse of October 4, 1884 , we are enabled to give Prof. Struve's times and position-angles for the stars that will be seen to be occulted by observers stationed at Edinburgh, Halifax, and Bath. A comparison of these tables will enable intending observers in other parts of England to form a sufficien'ly correct list for their own locality.

The fol owing ten stars, not included in the list given in NATURE for January 19, will be occulted as seen from Edinburgh :-

\begin{tabular}{|c|c|c|c|c|c|}
\hline Star's & R.A. & Decl. & Star's & R.A. & Decl. \\
\hline $\begin{array}{l}\text { No. } \\
\text { I03... I }\end{array}$ & & N. & $\begin{array}{l}\text { No. } \\
183 \ldots 13 \text { i }\end{array}$ & I $13^{\prime} 94$ & $8 \cdot 64 \mathrm{~N}$. \\
\hline $06 \ldots$ & 33.62 . & 16.68 & $206 \ldots$ & $2479 \ldots$ & 7.44 \\
\hline 7 . & $37.99 \ldots$ & 14.54 & $213 \ldots$ & $29.29 \ldots$ & 6.11 \\
\hline . & $4471 \ldots$ & 13.74 & $217 \ldots$ & $3 I \cdot 16 \ldots$ & $5 \cdot 36$ \\
\hline I... & $.5376 \ldots$ & 1484 & $228 \ldots$ & $34 \cdot 65$ & $6 \cdot 26$ \\
\hline
\end{tabular}

Star No. 106 is of mag. 9.3 ; No. $129,9.5$; No. 206 is of the roth magnitude; the others are all of the Irth magnitude.

EDINBURGh.

Lat. $=55^{\circ} 57^{\prime} 23^{\prime \prime}$; Long. $=3^{\circ} 10^{\prime} 54^{\prime \prime} \mathrm{W}$. Disappearances.

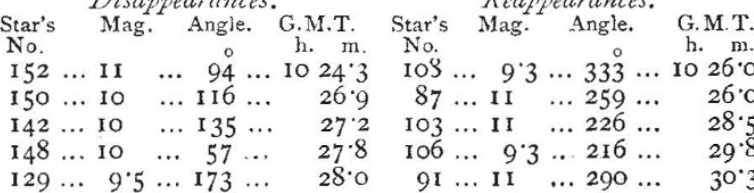

